\title{
Estado Nutricional e Sintomas Gastrointestinais em Pacientes Oncológicos Submetidos à Quimioterapia
}

doi: https://doi.org/10.32635/2176-9745.RBC.2021v67n2.1036

\author{
Nutritional Status and Gastrointestinal Symptoms in Oncology Patients Receiving Chemotherapy \\ Estado Nutricional y Síntomas Gastrointestinales en Pacientes con Cáncer Sometidos a Quimioterapia
}

\author{
Larissa Casari'; Vera Lúcia Ferreira da Silva²; Otávio Augusto Moura Fernandes³ ${ }^{3}$ Laura Moreira Goularte ${ }^{4}$; Deize Elizandra Vieira \\ Fanka5; Shirley Sousa de Oliveira ${ }^{6}$; Karina Sanches Machado d'Almeida ${ }^{7}$; Anne y Castro Marques ${ }^{8}$
}

\begin{abstract}
RESUM0
Introduçáo: $\mathrm{O}$ estado nutricional do paciente oncológico apresenta grande variação ao longo da doença, além de efeitos adversos relacionados ao trato gastrointestinal serem comuns durante o tratamento quimioterápico. Objetivo: Avaliar o estado nutricional e a presença de sintomas gastrointestinais em pacientes oncológicos submetidos à quimioterapia. Método: Estudo transversal com pacientes em tratamento no setor de Quimioterapia do Hospital Escola da Universidade Federal de Pelotas (UFPel), RS, no período de abril a julho de 2019. Dados sociodemográficos e questôes relacionadas à doença foram obtidos por meio de um questionário. Para avaliaçáo do estado nutricional e dos sintomas gastrointestinais, utilizou-se a avaliaçáo subjetiva global produzida pelo paciente. A análise dos dados foi realizada por meio de análise descritiva, e a associação entre variáveis categóricas, verificada pelo teste qui-quadrado de Pearson $(\mathrm{p}<0,05)$. Resultados: Foram avaliados 101 pacientes, com média de idade de 58,6 anos e maior prevalência de indivíduos do sexo feminino (58,4\%), de cor branca $(77,2 \%)$, casados $(53,0 \%)$, pertencentes à classe $\mathrm{C}(50,4 \%)$. Encontrou-se maior prevalência dos cânceres do trato gastrointestinal $(34,6 \%)$ e mama (27,8\%). A maioria dos pacientes foi classificada como bem nutrido (66,3\%), enquanto saciedade precoce $(56,0 \%)$, xerostomia $(54,0 \%)$, inapetência $(42,0 \%)$ e náusea $(37,0 \%)$ foram os sintomas mais citados. Não foi encontrada associação estatística entre o estado nutricional e qualquer um dos sintomas gastrointestinais. Conclusáo: $\mathrm{O}$ estado nutricional da maioria dos pacientes foi classificado como bem nutrido, mas necessitando de atençáo para a ocorrência dos sintomas gastrointestinais.

Palavras-chave: Antineoplásicos/efeitos adversos; Neoplasias; Avaliação Nutricional; Estado Nutricional; Efeitos Colaterais e Reaçôes Adversas Relacionados a Medicamentos.
\end{abstract}

\section{ABSTRACT}

Introduction: The nutritional status of oncologic patients varies greatly throughout the disease, further to gastrointestinal tract related adverse effects that are common during chemotherapy treatment. Objective: Evaluate the nutritional status and the presence of gastrointestinal symptoms in oncologic patients undergoing chemotherapy. Method: Cross-sectional study with patients in treatment in the Chemotherapy Unit of the Hospital School of Pelotas Federal University (UFPel), RS, from April to July 2019. Sociodemographic data and questions related to the disease were obtained through a questionnaire. To assess the nutritional status and gastrointestinal symptoms the Patient-Generated Subjective Global Assessment was utilized. Data analysis was performed through descriptive analysis, and the association between categorical variables was verified by Pearson's chi-square test $(\mathrm{p}<0.05)$. Results: A total of one hundred one patients was evaluated, mean age of 58.6 years, with higher prevalence of females $(58.4 \%)$, Caucasian $(77.2 \%)$, married $(53.0 \%)$, belonging to class C (50.4\%). Higher prevalence of gastrointestinal tract (34.6\%) and breast $(27.8 \%)$ cancer was found. Most patients were classified as well-nourished (66.3\%), while early satiety $(56.0 \%)$, xerostomia $(54.0 \%)$, loss of apetite $(42.0 \%)$, and nausea $(37.0 \%)$ were the symptoms most cited. No statistical association was encountered between nutritional status and any of the gastrointestinal symptoms. Conclusion: The nutritional status of most patients was classified as well nourished, but attention is needed for the occurrence of gastrointestinal symptoms.

Key words: Antineoplastic Agents/adverse effects; Neoplasms; Nutrition Assessment; Nutritional Status; Drug-Related Side Effects and Adverse Reactions.

\section{RESUMEN}

Introducción: El estado nutricional del paciente oncológico presenta variacones durante la enfermedad, además de los efectos adversos relacionados con el tracto gastrointestinal comunes durante la quimioterapia. Objetivo: Evaluar el estado nutricional y la presencia de síntomas gastrointestinales en pacientes con cáncer sometidos a quimioterapia. Método: Estudio transversal con pacientes tratados en el sector de quimioterapia del Hospital Escuela de la Universidad Federal de Pelotas (UFPel), RS, de abril a julio de 2019. Se obtuvieron datos sociodemográficos y relacionados con la enfermedad a través de un cuestionario. Para evaluar el estado nutricional y los síntomas gastrointestinales se utilizó la Evaluación Subjetiva Global Producida por el Paciente. Las análisis de los datos se realizó mediante análisis descriptiva, y la asociación entre variables categóricas se verificó mediante la prueba de chicuadrado de Pearson ( $\mathrm{p}<0,05)$. Resultados: Se evaluaron 101 pacientes, con edad media de 58,6 ańos, mayor prevalencia de mujeres $(58,4 \%)$, blancos $(77,2 \%)$, casados $(53,0 \%)$, pertenecientes a la clase C $(50,4 \%)$. Se encontró una mayor prevalencia de cánceres del tracto gastrointestinal $(34,6 \%)$ y de mama (27,8\%). La mayoría de los pacientes se clasificó como bien nutrido $(66,3 \%)$, mientras que saciedad temprana $(56,0 \%)$, boca seca $(54,0 \%)$, falta de apetito $(42,0 \%)$ y náuseas $(37,0 \%)$ fueron los síntomas más frecuentemente. No se encontró asociación estadística entre el estado nutricional y los síntomas gastrointestinales. Conclusión: El estado nutricional de la mayoría de los pacientes se clasificó como bien nutrido, pero necesita la atención a presencia de síntomas gastrointestinales.

Palabras clave: Antineoplásicos/efectos adversos; Neoplasias; Evaluación Nutricional; Estado Nutricional; Efectos Colaterales y Reacciones Adversas Relacionados con Medicamentos.

\footnotetext{
1-5,8Universidade Federal de Pelotas. Pelotas (RS), Brasil. E-mails: laaricasari@gmail.com; verinhaxavante1964@gmail.com; otavioaugustomofe@gmail.com; lauragoularte99@gmail.com; deise.fanka.nutricao@gmail.com; annezita@gmail.com. Orcid iD: https://orcid.org/0000-0002-4768-0424; Orcid iD: https://orcid. org/0000-0001-6918-5101; Orcid iD: https://orcid.org/0000-0003-4955-4357; Orcid iD: https://orcid.org/0000-0001-9710-1761; Orcid iD: https://orcid.org/00000001-7212-9434; Orcid iD: https://orcid.org/0000-0003-4905-6921

${ }^{6} \mathrm{Hospital}$ Universitário Polydoro Ernani de São Thiago. Florianópolis (SC), Brasil. E-mail: sschirleyoliveira@yahoo.com.br. Orcid iD: https://orcid.org/0000-0003-2976-1041 7Universidade Federal do Pampa (Unipampa). Itaqui (RS), Brasil. E-mail: karinasmdalmeida@unipampa.edu.br. Orcid iD: https://orcid.org/0000-0002-0439-3599 Endereço para correspondência: Larissa Casari. Faculdade de Nutrição da Universidade Federal de Pelotas. Rua Gomes Carneiro, 1 - Centro. Pelotas (RS), Brasil. CEP 96010-610. E-mail: laaricasari@gmail.com
} 


\section{INTRODUÇÃO}

Câncer é um conjunto de doenças que têm como característica o crescimento desordenado de células, incapazes de desenvolverem suas funções orgânicas, e que invadem órgáos e tecidos, atingindo outras regiōes do corpo $^{1}$. No Brasil, o câncer representa a terceira causa de morte, sendo superado somente pelas doenças circulatórias e por fatores externos. Os tipos de maior prevalência entre os brasileiros do sexo masculino são atualmente o câncer de próstata e os do aparelho respiratório e, entre as mulheres, os tumores de mama, cólon e reto e colo do útero. É válido destacar que no país o tipo de maior prevalência, em ambos os sexos, é o câncer não melanoma ${ }^{2}$.

$\mathrm{O}$ estado nutricional do paciente oncológico merece atenção especial, visto apresentar grande variação ao longo do tratamento e da doença. A desnutrição ocorre na maioria dos pacientes com câncer, sendo a maior causa de morbimortalidade entre esses indivíduos ${ }^{3}$. Em geral, os tumores que mais causam déficit nutricional são aqueles que estão localizados no trato gastrointestinal (pâncreas, esôfago e estômago), nas regiōes de cabeça e pescoço e no pulmãó .

Por outro lado, o tratamento pode induzir o ganho de peso, visto que os antineoplásicos, principalmente quando associados a glicocorticoides, podem gerar retenção hídrica e aumento de gordura corpórea ${ }^{5}$, como no caso do câncer de mama. Além disso, a dose do medicamento é baseada na área de superfície corporal e no peso, e tende a ser superestimada por não ter em conta fatores individuais, o que pode levar o paciente com baixo peso, obeso e/ou idoso à ocorrência de toxicidades ${ }^{6}$. Também é conhecida atualmente a associaçáo entre alguns tipos de câncer e obesidade, bem como a maior chance de recorrência da doença ${ }^{7,8}$.

Com o intuito de realizar uma avaliação nutricional adequada do paciente oncológico, indica-se a utilização da avaliação subjetiva global produzida pelo paciente (ASG-PPP), a qual possibilita uma maior sensibilidade para detectar desnutrição, além de identificar a presença ou não de alteraçóes gastrointestinais que impactam no estado nutricional dos pacientes, indicando a necessidade de intervençôes nutricionais precoces ${ }^{5,9}$.

Atualmente, uma das formas mais utilizadas de tratamento antineoplásico é a quimioterapia, que configura na administração de medicamentos por via oral ou endovenosa com o objetivo de exterminar as células malignas. Os antineoplásicos, no entanto, são incapazes de diferenciar o tecido normal e o neoplásico, podendo causar efeitos adversos em diferentes tecidos, principalmente naqueles com células de proliferação rápida ${ }^{10,11}$. $\mathrm{O}$ trato gastrointestinal, por possuir células com capacidade proliferativa intensa, acaba por sofrer com a toxicidade não específica dos antineoplásicos. Entre os efeitos adversos mais frequentes no trato gastrointestinal, estão: vômitos, náusea, mucosite oral, diarreia, má absorção de nutrientes, constipação intestinal, aversão alimentar e xerostomia ${ }^{5,6,12}$.

Conforme explanado anteriormente, o estado nutricional do paciente oncológico em tratamento quimioterápico pode sofrer grandes mudanças, sendo influenciado diretamente pelos efeitos tóxicos no trato gastrointestinal e repercutindo no seu prognóstico. Dessa forma, o presente estudo teve como objetivo avaliar o estado nutricional e a presença de sintomas gastrointestinais em pacientes oncológicos submetidos à quimioterapia.

\section{MÉTODO}

Estudo transversal, observacional, com pacientes em tratamento no setor de Quimioterapia do Hospital Escola da Universidade Federal de Pelotas (UFPel), RS, no período de abril a julho de 2019. Este trabalho faz parte de um projeto maior intitulado, "Hábitos alimentares e qualidade de vida de pacientes em quimioterapia", aprovado pelo Comitê de Ética em Pesquisa da Faculdade de Medicina da UFPel, sob o número 2.927.703. A coleta foi realizada apenas por um acadêmico do Curso de Nutrição da UFPel, previamente treinado, a fim de evitar vieses.

A amostra foi constituída por mulheres e homens com idade acima de 18 anos, a partir do segundo ciclo de quimioterapia, que aceitaram participar da pesquisa mediante assinatura do Termo de Consentimento Livre e Esclarecido (TCLE), durante a realização da aplicação dos quimioterápicos. Excluíram-se do estudo gestantes e pacientes incapazes de responder ao questionário.

Os dados foram coletados a partir de um questionário, previamente elaborado pelos autores e, a partir dessa ferramenta, obtiveram-se as informaçóes sociodemográficas de interesse no estudo (sexo, idade, cor da pele, estado civil, profissão), sendo a classe social definida de acordo com a Associação Brasileira de Empresas de Pesquisa ${ }^{13}$. Em relação ao estado de saúde, foram obtidas em prontuário as informaçôes sobre localização do tumor primário, presença ou não de metástase e de quimioterápicos em uso (posteriormente categorizados de acordo com a classe); já a presença de outras doenças crônicas foi informada pelo próprio paciente.

O estado nutricional dos sujeitos foi obtido a partir da ASG-PPP na versão em português, validada por Gonzalez et al. ${ }^{9}$. Trata-se de uma ferramenta específica para determinar o estado nutricional de pacientes com câncer (independentemente do tipo de tratamento), sendo um método que combina fatores como alteraçóes 
na ingestão de alimentos, mudanças de peso, ocorrência de sintomas gastrointestinais, mudanças físicas e funcionais do paciente. Para este estudo, foram consideradas as três categorias de estado nutricional: $\mathrm{A}=$ bem nutrido; $\mathrm{B}=$ moderadamente desnutrido ou suspeito de desnutrição; e $\mathrm{C}=$ gravemente desnutrido. A ASG-PPP também fornece um escore total, o qual pode ser utilizado para definir intervenções nutricionais específicas: escores de 0 a 1 indicam que náo há necessidade de intervenção nutricional no momento; 2 a 3 que há necessidade de educação do paciente e seus familiares pelo profissional de saúde, com intervenção farmacológica de acordo com os sintomas e exames laboratoriais, se adequado; 4 a 8 indicam que é necessário intervenção pelo nutricionista e outros profissionais; e escores acima de 9 que existe necessidade crítica de melhora no manuseio dos sintomas e/ou opçóes de intervenção nutricional ${ }^{9}$.

A ASG-PPP ainda contém um quadro específico sobre sintomas relacionados ao consumo alimentar, no qual o paciente deve apontar quais foram apresentados nas duas últimas semanas; para este estudo, foram considerados os sintomas gastrointestinais: náusea, vômito, diarreia, constipação, mucosite oral, xerostomia, inapetência e saciedade precoce.

Os dados foram digitados no programa Microsoft Excel e posteriormente revisados pelo pesquisador responsável, sendo realizada análise descritiva. A associação entre o estado nutricional (avaliação categórica) e os sintomas gastrointestinais foi verificada segundo o teste qui-quadrado de Pearson, com $\mathrm{p}<0,05$, no programa estatístico Statistical Package for the Social Sciences (SPSS) versão 25.0.

\section{RESULTADOS}

Foram convidados a participar deste estudo 116 sujeitos, dos quais, 15 recusaram ou não puderam responder, totalizando 101 pacientes. A amostra estudada apresentou média de idade de 58,6 $\pm 12,2$ anos e maior prevalência de indivíduos do sexo feminino, de cor branca, casados, pertencentes à classe $\mathrm{C}$ e aposentados, conforme pode ser observado na Tabela 1 .

Em relação aos dados sobre a doença (Tabela 2), observou-se maior prevalência dos cânceres primários localizados no trato gastrointestinal, na mama e no pulmão, e aproximadamente um terço dos pacientes tinha diagnóstico de metástase. A maioria dos sujeitos referiu não apresentar outras doenças crônicas; entre os que responderam afirmativamente, hipertensão e diabetes foram as mais relatadas. Em relação aos quimioterápicos, os mais utilizados foram os agentes alquilantes, seguido por protocolos contendo duas ou mais classes de quimioterápicos.
Tabela 1. Dados sociodemográficos de pacientes em tratamento quimioterápico de um hospital público em Pelotas, RS, 2019. N=101

\begin{tabular}{lcc}
\multicolumn{1}{c}{ Variável } & N & $\begin{array}{c}\text { \% ou média } \\
\text { (DP) }\end{array}$ \\
\hline Sexo & & \\
Feminino & 59 & 58,4 \\
Masculino & 42 & 41,6 \\
Idade & & \\
Idade (anos) & \\
Cor da pele & 58,6 & $(12,2)$ \\
Brancos & & \\
Não brancos & 78 & 77,2 \\
Estado civil (n=100) & 23 & 22,8 \\
Solteiro & & \\
Casado & 23 & 23,0 \\
Divorciado & 53 & 53,0 \\
Viúvo & 12 & 12,0 \\
Classe social & 12 & 12,0 \\
Classe A & & \\
Classes B1 e B2 & 4 & 4,0 \\
Classes C1 e C2 & 24 & 23,8 \\
Classes D/E & 51 & 50,4 \\
Profissáo (n=100) & 22 & 21,8 \\
Aposentado(a) & & \\
Dona de casa & 32 & 32,0 \\
Doméstica/cozinheiro(a) & 12 & 12,0 \\
Agricultor/pecuarista & 7 & 12,0 \\
Professor(a) & 5 & 7,0 \\
Área da saúde & 3 & 5,0 \\
Outros & 29 & 29,0 \\
\hline & & \\
\hline
\end{tabular}

Legendas: DP: Desvio-padrão; * Idade mínima: 24 anos; Idade máxima: 91 anos.

Os dados referentes ao estado nutricional e aos sintomas gastrointestinais são apresentados na Tabela 3 . Considerando-se o estado nutricional pela ASG-PPP, a maioria dos pacientes em quimioterapia foi classificada como bem nutrida. Quando avaliado o escore da ASG-PPP, o escore médio foi de 13 pontos (desvio-padrão de 7,33), indicando necessidade de conduta para melhora dos sintomas e/ou intervenção nutricional. Em relação aos sintomas gastrointestinais, os mais prevalentes foram saciedade precoce, xerostomia, inapetência e náusea.

Não foi encontrada associação entre o estado nutricional (avaliação global) e qualquer um dos sintomas gastrointestinais $(\mathrm{p}<0,05)$. Também não foi encontrada associação entre os quimioterápicos utilizados e o estado nutricional dos pacientes, tanto se considerada a classificação global de estado nutricional $(\mathrm{p}=0,528)$ como o escore da ASGP-PPP ( $\mathrm{p}=0,823)$. 
Tabela 2. Dados sobre a doença de pacientes em tratamento quimioterápico de um hospital público em Pelotas, RS, 2019. N=101

\begin{tabular}{|c|c|c|}
\hline Variável & $\mathbf{N}$ & $\%$ \\
\hline \multicolumn{3}{|l|}{ Local do tumor inicial } \\
\hline Trato gastrointestinal & 35 & 34,6 \\
\hline Intestino & 22 & 21,8 \\
\hline Esôfago/estômago & 7 & 6,9 \\
\hline Fígado/pâncreas & 6 & 5,9 \\
\hline \multicolumn{3}{|l|}{ Outros locais } \\
\hline Mama & 28 & 27,8 \\
\hline Pulmão & 10 & 9,9 \\
\hline Ginecológico & 10 & 9,9 \\
\hline Próstata/testículo & 7 & 6,9 \\
\hline Pele & 3 & 3,0 \\
\hline Cabeça/pescoço & 2 & 2,0 \\
\hline Outros & 6 & 5,9 \\
\hline \multicolumn{3}{|l|}{ Diagnóstico de metóstase } \\
\hline Não & 69 & 68.3 \\
\hline Sim & 32 & 31,7 \\
\hline \multicolumn{3}{|l|}{ Doenças associadas } \\
\hline Hipertensão & 25 & 24,8 \\
\hline Diabetes & 8 & 7,9 \\
\hline Hipertensão e diabetes & 4 & 4,0 \\
\hline Outras & 4 & 4,0 \\
\hline Sem comorbidades & 60 & 59,4 \\
\hline \multicolumn{3}{|l|}{$\begin{array}{l}\text { Quimioterápicos em uso } \\
\text { (classes)* }\end{array}$} \\
\hline Agentes alquilantes & 26 & 26,0 \\
\hline Derivados de vegetais & 14 & 14,0 \\
\hline Bifosfonatos & 10 & 10,0 \\
\hline Anticorpos monoclonais & 6 & 6,0 \\
\hline $\begin{array}{l}\text { Compostos de coordenação de } \\
\text { platina }\end{array}$ & 4 & 4,0 \\
\hline Análogos de camptotecina & 1 & 1,0 \\
\hline $\begin{array}{l}\text { Derivados vegetais + agentes } \\
\text { alquilantes }\end{array}$ & 13 & 13,0 \\
\hline $\begin{array}{l}\text { Agentes alquilantes }+ \\
\text { antibióticos antitumorais }\end{array}$ & 6 & 6,0 \\
\hline $\begin{array}{l}\text { Protocolos com duas ou mais } \\
\text { classes }\end{array}$ & 20 & 20,0 \\
\hline
\end{tabular}

Legenda: ${ }^{*} \mathrm{~N}=100$ (houve perda de uma resposta).

\section{DISCUSSÃO}

No presente estudo, foi encontrada maior proporçáo de pacientes do sexo feminino, casados e pertencentes à classe C. Dados semelhantes foram encontrados por Capelari e Ceni ${ }^{14}$ em um Centro de Alta Complexidade
Tabela 3. Estado nutricional e sintomas gastrointestinais de pacientes em tratamento quimioterápico de um hospital público em Pelotas, $\mathrm{RS}, 2019 . \mathrm{N}=101$

\begin{tabular}{lcc}
\hline \multicolumn{1}{c}{ Variável } & N & $\%$ \\
\hline Estado nutricional (ASG-PPP) & & \\
A & 67 & 66,3 \\
B & 23 & 22,8 \\
C & 11 & 10,9 \\
Sintomas gastrointestinais* & & \\
Inapetência & 42 & 42,0 \\
Náusea & 37 & 37,0 \\
Vômito & 9 & 9,0 \\
Constipação & 28 & 28,0 \\
Diarreia & 27 & 27,0 \\
Mucosite & 26 & 26,0 \\
Xerostomia & 54 & 54,0 \\
Saciedade precoce & 56 & 56,0 \\
\hline
\end{tabular}

Legendas: A: bem nutrido; B: moderadamente desnutrido ou suspeita de desnutrição; C: gravemente desnutrido. ${ }^{*}$ Houve uma perda nesta resposta $(\mathrm{n}=100)$. Os sintomas gastrointestinais ultrapassam $100 \%$, pois o mesmo paciente pode ter referido mais de um sintoma.

em Oncologia localizado no Noroeste do RS, no qual, dos 100 indivíduos avaliados, 56,0\% eram mulheres; e $60,7 \%$ das mulheres e $79,5 \%$ dos homens eram casados. Quanto à classe social, já era esperado neste estudo maior público de classe média-baixa, por se tratar de um centro quimioterápico com atendimentos exclusivamente realizados pelo Sistema Único de Saúde.

Em relação à localização do tumor primário, nesta amostra encontrou-se maior prevalência dos cânceres de trato gastrointestinal e de mama. Em um estudo realizado no mesmo local, há aproximadamente sete anos, no qual avaliaram-se 83 pacientes, os cânceres do trato gastrointestinal também foram os mais prevalentes ${ }^{15}$. Por sua vez, o câncer de mama é a principal neoplasia que acomete as mulheres em nível nacional, tendo altas taxas de mortalidade ${ }^{2}$.

Quanto à presença de doenças associadas, cerca de um terço dos pacientes referiram ter hipertensão el ou diabetes. Dados semelhantes foram encontrados por Santos et al. ${ }^{16}$, que avaliaram 20 pacientes do sexo feminino diagnosticadas com câncer de mama, em tratamento quimioterápico, em um hospital de Recife, PE. É importante ressaltar que a presença de múltiplas comorbidades em pacientes oncológicos afeta frequentemente as decisóes de tratamento do câncer, sendo mais propensos a receber uma dose reduzida, bem como não acabando o tratamento quando iniciado, aumentando os riscos de mortalidade e eventos adversos ${ }^{17}$. 
Com relação à terapêutica, existem diferentes protocolos de tratamento quimioterápico estabelecidos de acordo com a especificidade da doença e das condiçóes de saúde do paciente ${ }^{11}$. Cerca de um quarto dos sujeitos deste estudo fazia uso de agentes alquilantes, os quais causam modificaçôes nas cadeias de DNA, contendo sua replicação. Esses quimioterápicos são bastante utilizados no tratamento dos cânceres de mama, sarcoma e mielomas, e apresentam, entre seus efeitos adversos, toxicidade às mucosas (incluindo do trato gastrointestinal) ${ }^{11}$.

Acerca do estado nutricional, a maioria dos pacientes foi classificada, segundo a ASG-PPP, como bem nutrida. Tal resultado mostra-se positivo, uma vez que a desnutrição traz como consequência menor qualidade de vida, diminuiçấo da tolerância ao tratamento e de sobrevida, maior chance de infecção e aumento dos custos ao sistema de saúde ${ }^{14}$. É válido ressaltar, no entanto, que a ASG-PPP também mostrou um escore médio indicando a necessidade urgente de conduta para aliviar os sintomas e/ou intervenção nutricional. Mesmo que estejam atualmente bem nutridos, a persistência dos sintomas gastrointestinais é fator de risco para a desnutrição e prejuízos a ela relacionados.

Mudanças no peso e na composição corporal em pacientes oncológicos são associadas aos tratamentos quimioterápicos, à hormonioterapia e à duraçâo deles. Em mulheres com câncer de mama, a diminuição de massa magra e o aumento da massa gorda (obesidade sarcopênica) são associadas à recidiva da doença e a outras comorbidades ${ }^{8}$. A prevenção de ganho de peso durante e após o tratamento quimioterápico tem o potencial de diminuir as taxas de mortalidade, pois o ganho de peso ativa os hormônios metabólicos (leptina e adiponectina), cujos mecanismos inibem a apoptose e promovem o crescimento celular, desencadeando um novo tumor ou favorecendo sua progressão ${ }^{18}$.

Apesar dos avanços da quimioterapia, ainda é comum que pacientes em tratamento cursem em risco nutricional ${ }^{19}$. Os quimioterápicos agem principalmente contra as células de rápida proliferação do epitélio gastrointestinal, tornando-o extremamente vulnerável à sua toxicidade, que se manifesta na forma de sintomas. Portanto, quaisquer alteraçóes fisiológicas, estruturais, bioquímicas ou farmacológicas nessas mucosas podem agravar o estado nutricional do paciente ${ }^{12,20}$. Entre os sintomas gastrointestinais, os mais citados neste estudo foram: empachamento $(56,0 \%)$, xerostomia $(54,0 \%)$, inapetência $(42,0 \%)$ e náusea $(37,0 \%)$. Contudo, outros estudos observaram diferentes sintomas como os mais prevalentes: constipação e diarreia $(58,3 \%)^{16} \mathrm{e}$ náusea $(47,5 \%)^{21}$. Ressalta-se ainda que, além dos efeitos adversos oriundos dos quimioterápicos, quase $35,0 \%$ dos participantes deste estudo apresentavam câncer no trato gastrointestinal, fator este que também contribuiu para o surgimento de sintomas relacionados ao sistema digestivo.

A xerostomia, um dos sintomas mais relatados pelos pacientes, também foi observada em um estudo dirigido por Mercadante et al. ${ }^{22}$ com 669 pacientes, dos quais, $40,4 \%$ mencionaram o sintoma. O terceiro sintoma mais citado no estudo foi inapetência. Corroborando este achado, Galindo et al. ${ }^{23}$ ( $\left.\mathrm{n}=128\right)$ observaram que $61,8 \%$ dos pacientes apresentavam alterações de apetite, entre os quais, $31,3 \%$ apresentavam inapetência e 13,3\% saciedade precoce.

No presente estudo, não foi encontrada associação significativa entre o estado nutricional e os sintomas gastrointestinais. Saragiotto ${ }^{24}$, em um estudo longitudinal retrospectivo no Hospital PUC-Campinas, cuja população era formada por adultos e idosos de ambos os sexos e portadores de câncer em tratamento quimioterápico, também verificou a ausência de diferenças estatisticamente significativas entre as variáveis sintomas gastrointestinais e estado nutricional.

Similarmente, não foi encontrada associação entre os quimioterápicos utilizados e o estado nutricional dos pacientes, tanto se considerada a classificação global de estado nutricional $(\mathrm{p}=0,528)$ como o escore da ASGP-PPP ( $\mathrm{p}=0,823)$. Ciclofosfamida, isofosfamida, cisplatina e carboplatina são os exemplos mais comuns de agentes alquilantes ${ }^{11}$. Em um estudo de revisão ${ }^{12}$, altas doses de cisplatina, ciclofosfamida, carboplatina e outros agentes possuem alto potencial emetogênico, podendo ocasionar náuseas e vômitos e, consequentemente, perda de apetite, mesmo após um dia do tratamento antineoplásicos.

O papel da nutriçáo na redução das morbidades e dos sintomas relacionados ao tratamento é vital. A triagem nutricional deve ser iniciada no momento do diagnóstico, não somente com o intuito de identificar desnutrição, mas também o ganho de peso não intencional. Intervençôes nutricionais precoces têm sido associadas a benefícios aos pacientes, como a prevenção de atrasos no tratamento, que ocorrem em razão de sintomas graves ${ }^{25}$.

Considera-se como uma das limitaçóes deste trabalho o baixo número amostral, quando comparado a outros estudos existentes com o mesmo público. Em contrapartida, ressalta-se o cuidado metodológico, visto que cada pesquisador se dedicou exclusivamente a uma única etapa, na tentativa de padronizar a coleta e a análise dos dados.

\section{CONCLUSÃO}

Neste estudo, o estado nutricional da maioria dos pacientes oncológicos em tratamento quimioterápico 
foi classificado como bem nutrido, mas necessitando de atenção para a ocorrência dos sintomas gastrointestinais. Entre os sintomas com maior prevalência, destacaram-se: saciedade precoce, xerostomia, inapetência e náusea. No entanto, não foi encontrada associação significativa entre o estado nutricional e os sintomas gastrointestinais.

É importante que estudos futuros investiguem o estado nutricional, relacionando sintomas de toxicidade quimioterápica com variaçóes de peso, tipo de tratamento, estadiamento da doença e localizaçáo do tumor, uma vez que o ganho de peso e a desnutrição em indivíduos com câncer merecem atenção.

\section{CONTRIBUIÇÕES}

Larissa Casari e Anne y Castro Marques contribuíram na concepção e/ou no planejamento do estudo; na obtençáo, análise e interpretação dos dados; assim como na redação e revisáo crítica. Vera Lúcia Ferreira da Silva, Otávio Augusto Moura Fernandes, Laura Moreira Goularte, Deize Elizandra Vieira Fanka, Shirley Sousa de Oliveira e Karina Sanches Machado d'Almeida contribuíram na concepçáo e/ou no planejamento do estudo; na obtenção, análise e interpretação dos dados. Todos os autores aprovaram a versão final a ser publicada.

\section{DECLARAÇÃO DE CONFLITO DE INTERESSES}

Nada a declarar.

\section{FONTES DE FINANCIAMENTO}

Não há.

\section{REFERÊNCIAS}

1. Descritores em Ciências da Saúde: DeCS [Internet]. ed. 2017. São Paulo (SP): BIREME / OPAS / OMS; 2017 [atualizado 2017 maio; acesso 2019 abr 4]. Disponível em: http://decs.bvsalud.org

2. Instituto Nacional de Câncer Jose Alencar Gomes da Silva [Internet]. Rio de Janeiro: INCA; [data desconhecida]. Causas e prevenção: estatísticas de câncer; [acesso 2019 out 29]. Disponível em: https://www.inca.gov.br/ numeros-de-cancer

3. Cutsema EV, Arends J. The causes and consequences of cancer-associated malnutrition. Eur J Oncol Nurs. 2005;9(Suppl 2):S51-S63. doi: https://doi. org/10.1016/j.ejon.2005.09.007

4. Brito LF, Silva LS, Fernandes DD, et al. Perfil nutricional de pacientes com câncer assistidos pela casa de acolhimento ao paciente oncológico do sudoeste da Bahia.
Rev Bras Cancerol. 2012;58(2):163-171. doi: https://doi. org/10.32635/2176-9745.RBC.2012v58n2.615

5. Soares BLM, Santos DRL, Carneiro ICLM, et al. Alteraçóes gastrintestinais e ponderais em pacientes submetidos à quimioterapia. Rev Bras Nutr Clin. 2013;28(2):103-6.

6. Rocha IMG. Toxicidade em pacientes com câncer no trato gastrointestinal durante a quimioterapia: associaçôes com sarcopenia e caquexia [dissertaçáo]. Rio Grande do Norte (RN): Centro de Ciências da Saúde, Universidade Federal do Rio Grande do Norte; 2018.

7. Souza CLS. Peso, composição corporal e distribuição de gordura corporal de mulheres em quimioterapia adjuvante para o câncer de mama: um estudo de acompanhamento [dissertação]. Salvador (BA): Escola de Nutrição, Universidade Federal da Bahia; 2012.

8. Pedersen B, Delmar C, Bendtsen MD, et al. Changes in weight and body composition among women with breast cancer during and after adjuvant treatment: a prospective follow-up study. Cancer Nurs. 2017;40(5):369-76. doi: https://doi.org/10.1097/NCC.0000000000000426

9. Gonzalez MC, Borges LR, Silveira DH, et al. Validação da versão em português da avaliação subjetiva global produzida pelo paciente. Rev Bras Nutr Clin. 2010;25(2):102-8.

10. Silva FCM, Comarella L. Efeitos adversos associados à quimioterapia antineoplásica: levantamento realizado com pacientes de um hospital do estado do Paraná. Rev Uniandrade. 2013;14(3):263-77. doi: https:// doi.org/10.18024/1519-5694/revuniandrade. v14n3p263-277

11. Andrade M, Silva SR. Administração de quimioterápicos: uma proposta de protocolo de enfermagem. Rev Bras Enferm. 2007;60(3):331-5. doi: https://doi. org/10.1590/S0034-71672007000300016

12. Mitchell EP. Gastrointestinal toxicity of chemotherapeutic agents. Semin Oncol. 2006;33(1):106-20. doi: https:// doi.org/10.1053/j.seminoncol.2005.12.001

13. Associação Brasileira de Empresas de Pesquisa. Alteraçóes na aplicação do Critério Brasil [Internet]. [Sáo Paulo]: ABEP; 2018 abr [acesso 2019 nov 24]. Disponível em: http://www.abep.org/criterioBr/01_cceb_2018.pdf

14. Capelari P, Ceni GC. Comportamento alimentar e perfil nutricional de pacientes oncológicos em tratamento quimioterápico. Demetra. 2018;13(1):223-240. doi: https://doi.org/10.12957/demetra.2018.30309

15. Colling C, Duval PA, Silveira DH. Pacientes submetidos à quimioterapia: avaliação nutricional prévia. Rev Bras Cancerol. 2012;58(4):611-7. doi: https://doi. org/10.32635/2176-9745.RBC.2012v58n4.563

16. Santos EMC, Silva LML, Santos EMC, et al. Associaçáo entre o estado nutricional e a presença de toxicidade gastrointestinal em pacientes com câncer de mama. Braspen J. 2018;33(1):9-14. 
17. Williams GR, Deal AM, Lund JL, et al. Patientreported comorbidity and survival in older adults with cancer. Oncologist. 2018;23(4):433-9. doi: https://doi. org/10.1634/theoncologist.2017-0404

18. Playdon MC, Bracken MB, Sanft TB, et al. Weight gain after breast cancer diagnosis and all-cause mortality: systematic review and meta-analysis. J Natl Cancer Inst. 2015;107(12):djv275. doi: https://doi.org/10.1093/ jnci/djv275

19. Hackbarth L, Machado J. Estado nutricional de pacientes em tratamento de câncer gastrointestinal. Rev Bras Nutr Clin. 2015;30(4):271-275.

20. Nolden A, Joseph PV, Kober KM, et al. Co-occurring gastrointestinal symptoms are associated with taste changes in oncology patients receiving chemotherapy. J Pain Symptom Manage. 2019;58(5):756-65. doi: https:// doi.org/10.1016/j.jpainsymman.2019.07.016

21. Singh KP, Kober KM, Dhruva AA, et al. Risk factors associated with chemotherapy-induced nausea in the week before the next cycle and impact of nausea on quality of life outcomes. J Pain Symptom Manage. 2018;56(3):352-62. doi: https://doi.org/10.1016/j. jpainsymman.2018.05.019

22. Mercadante S, Aielli F, Adile C, et al. Prevalence of oral mucositis, dry mouth, and dysphagia in advanced cancer patients. Support Care Cancer. 2015;23(11):3249-55. doi: https://doi.org/10.1007/s00520-015-2720-y

23. Galindo DEB, Vidal-Casariego A, Calleja-Fernández A, et al. Appetite disorders in cancer patients: Impact on nutritional status and quality of life. Appetite. 2017;114:23-27. doi: https://doi.org/10.1016/j. appet.2017.03.020

24. Saragiotto L. Efeitos colaterais da quimioterapia durante a evolução nutricional de pacientes oncológicos em acompanhamento ambulatorial [dissertação]. Campinas: Pontifícia Universidade Católica de Campinas; 2018.

25. Wilkes PA, Allen DH. Nutrition Care: managing symptoms from cancer. J Nurse Pract. 2018;14(4):26775. doi: https://doi.org/10.1016/j.nurpra.2018.01.011 